\title{
Tackling Privacy on Social Media
}

\author{
C.P. Kedia ${ }^{1}$, Suraj Tavare ${ }^{2}$, Vikram Karkande ${ }^{3}$, Vivek Sonawane ${ }^{4}$, Pranjul Avi $^{5}$ \\ Asst. Professor, Comp Department, TAE, Pune, India ${ }^{1}$ \\ Student, Comp Department, TAE, Pune, India ${ }^{2,3,4,5}$
}

\begin{abstract}
With the increasing volume of images users share through social sites, maintaining privacy has become a major problem, as demonstrated by a recent wave of publicized incidents where users inadvertently shared personal information. Social Network is an emerging E-service for content sharing sites (CSS). It is emerging service which provides a reliable communication, through this communication a new attack ground for data hackers; they can easily misuses the data through these media. Some users over CSS affects users privacy on their personal contents, where some users keep on sending unwanted comments and messages by taking advantage of the users' inherent trust in their relationship network. To discussing this we focus on the issue to protect user Images on social site. Images are travelling on social site so we decide policy how image will be travel on social network. We consider to decide how to decide policy where own image become protective. This proposes a privacy policy prediction and access restrictions along with blocking scheme for social sites using data mining techniques. Toward addressing this need, we propose a novel system to help users compose privacy settings for their images. To deal with this dilemma, our mechanism attempts to utilize users' private photos to design a personalized FR system specifically trained to differentiate possible photo co-owners without leaking their privacy.
\end{abstract}

Keywords: online social networks, FR system, open social, photo privacy.

\section{INTRODUCTION}

Social media is very powerful tool to communicate with each other, user can communicate with social site to exchange idea, emotion, information, happiness. Now every user are connect to each other, there are very high volume which are connect with each other using different sites.

Social media is the two way communication in Web and it means to communicate, share, and interact with an individual or with a large audience. Social networking websites are the most famous websites on the Internet and millions of people use them every day to engage and connect with other people. Number of web site like Facebook, Twitter, etc users are use to communicate, connected with each other, user can upload, post, tweet, download images video and performing number of action.

An overview was led to concentrate the adequacy of the existing counter measure of un-tagging and demonstrates that this counter measure is a long way from attractive users are stressing about culpable their companions when untagging. Therefore, they give an instrument to empower users to limit others from seeing their photos when posted as a correlative methodology to ensure protection.

Sadly, on most current OSNs, clients have no control over the data showing up outside their profile page. Be that as it may, in current OSNs, when posting a photograph, a client is not required to request consents of different clients showing up in the photograph. Fundamentally, in our proposed one-against-one system a client needs to build up classifiers between self, companion and companion, companion otherwise called the two circles in Algorithm.

\section{LITERATURE SURVEY}

- Collaborative face recognition for improved face annotation in personal photo collections shared on online social networks

Using face annotation for effective management of personal photos in online social networks (OSNs) is currently of considerable practical interest. In this paper, we propose a novel collaborative face recognition (FR) framework, improving the accuracy of face annotation by effectively making use of multiple FR engines available in an OSN. Our collaborative FR framework consists of two major parts: selection of FR engines and merging (or fusion) of multiple FR results. The selection of FR engines aims at determining a set of personalized FR engines that are suitable for recognizing query face images belonging to a particular member of the OSN.

- $\quad$ A collaborative face recognition framework on a social network platform.

Face recognition has many useful applications spanning surveillance, law enforcement, information security, smart card and entertainment technologies. Very recently, a learning based face recognition system is also seen to be applied to web platform combining face recognition and web service. However, many existing methods which focused on 
recognition accuracy cannot cope with the new social network platform because the adopted static learning approach is not adaptive to daily updated photographs among the massive number of users.

\section{- $\quad$ Toward Large-Scale Face Recognition Using Social Network Context}

Personal photographs are being captured in digital form at an accelerating rate, and our computational tools for searching, browsing, and sharing these photos are struggling to keep pace. One promising approach is automatic face recognition, which would allow photos to be organized by the identities of the individuals they contain. However, achieving accurate recognition at the scale of the Web requires discriminating among hundreds of millions of individuals and would seem to be a daunting task. This paper argues that social network context may be the key for large-scale face recognition to succeed. Many personal photographs are shared on the Web through online social network sites, and we can leverage the resources and structure of such social networks to improve face recognition rates on the images shared.

\section{PROPOSED SYSTEM}

In online social network a large portion of the people transfer photograph without the worry of the protection spillage and security. This application is created to save protection of the clients and make the framework more secure. The framework is made secure by creating OTP at the season of login stage and furthermore when clients transfer the photograph. The application recognizes and trims the face from the transferred photograph and produce an arrangement of prepare pictures. The framework should be prepared by more number of pictures. The FR framework is produced utilizing LBPH algorithm which perceives the people show in the photograph and hence advises them about the photograph posting and indeed, even make them effectively partake in the posting activity. Preserving security investigation have given proposed conspire that can adequately accomplish classification of photographs transferred.

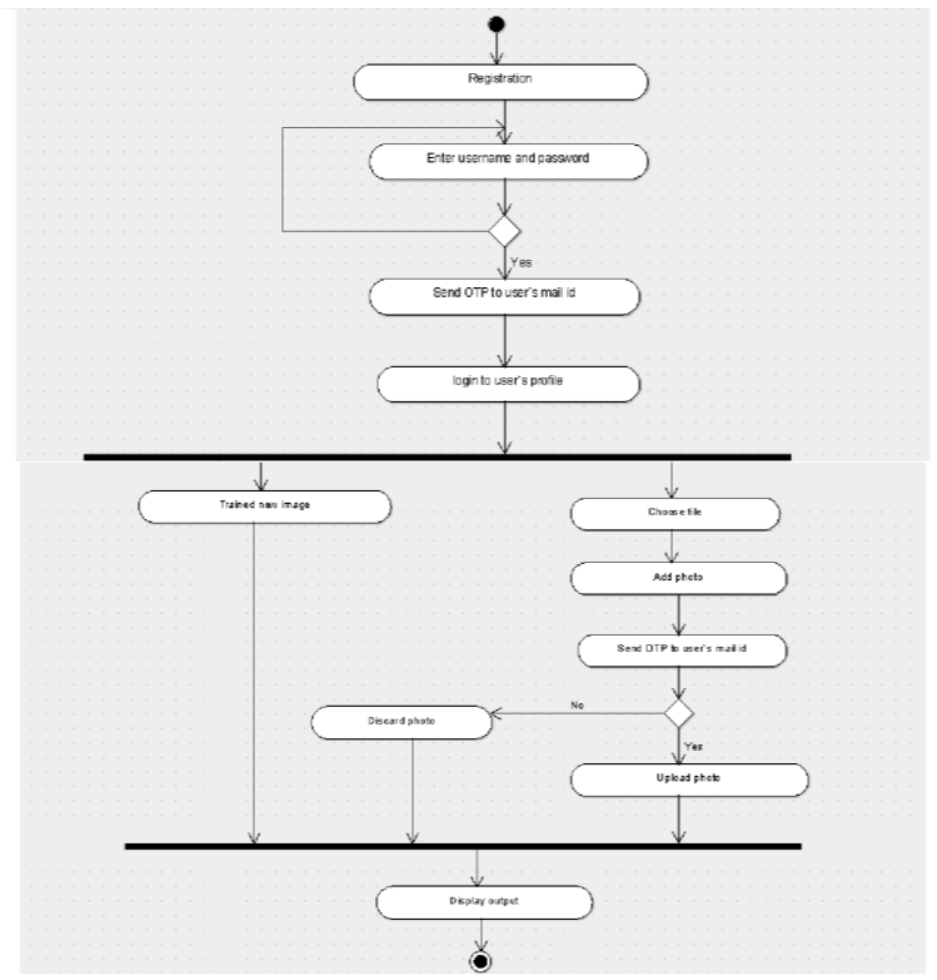

Flow of project

\section{ADVANTAGE OF PROPOSED SYSTEM}

- User friendly system

- Make user`s account secured

- To reduce the computational complexity and protect the private data of user.

- It is very efficient than existing system

- The system can reduce the privacy leakage 


\section{IJARCCE}

\section{CONCLUSION}

Photograph sharing is a standout amongst the most prominent elements in online social networks, for example, Facebook. Sadly, thoughtless photograph posting may uncover protection of people in a posted photograph. To control the protection spillage, we proposed to empower people possibly in a photograph to give the consents before posting a co-photograph. We planned a protection safeguarding FR framework to recognize people in a co-photograph. The proposed framework is highlighted with low calculation cost and privacy of the preparation set. Hypothetical investigation and trials were led to show viability and proficiency of the proposed conspire.

\section{REFERENCES}

[1] I. Altman. Privacy regulation: Culturally universal or culturally specific? Journal of Social Issues, 33(3):66-84, 1977.

[2] A. Besmer and H. Richter Lipford. Moving beyond untagging: photo privacy in a tagged world. In Proceedings of the SIGCHI Conference on Human Factors in Computing Systems, CHI '10, pages 1563-1572, New York, NY, USA, 2010. ACM.

[3] S. Boyd, N. Parikh, E. Chu, B. Peleato, and J. Eckstein. Distributed optimization and statistical learning via the alternating direction method of multipliers. Found. Trends Mach. Learn., 3(1):1-122, Jan. 2011.

[4] B. Carminati, E. Ferrari, and A. Perego. Rule-based access control for social networks. In R. Meersman, Z. Tari, and P. Herrero, editors, On the Move to Meaningful Internet Systems 2006: OTM 2006 Workshops, volume 4278 of Lecture Notes in Computer Science, pages 1734-1744. Springer Berlin Heidelberg, 2006.

[5] J. Y. Choi, W. De Neve, K. Plataniotis, and Y.-M. Ro. Collaborative face recognition for improved face annotation in personal photo collections shared on online social networks. Multimedia, IEEE Transactions on, 13(1):14-28, 2011.

[6] K. Choi, H. Byun, and K.-A. Toh. A collaborative face recognition framework on a social network platform. In Automatic Face Gesture Recognition, 2008. FG '08. 8th IEEE International Conference on, pages 1-6, 2008.

[7] K.-B. Duan and S. S. Keerthi. Which is the best multiclass svmmethod? an empirical study. In Proceedings of the 6th international conference on Multiple Classifier Systems, MCS'05, pages 278-285, Berlin, Heidelberg, 2005. Springer-Verlag.

[8] P. A. Forero, A. Cano, and G. B. Giannakis. Consensus-based distributed support vector machines. J. Mach. Learn. Res., 99:1663- 1707, August 2010 